\title{
Coronary computed tomography angiography equals invasive angiography for the prediction of coronary revascularization
}

\author{
Mariusz Dębski, Mariusz Kruk, Sebastian Bujak, Zofia Dzielińska, Marcin Demkow, Cezary Kępka \\ Department of Coronary and Structural Heart Diseases, The Cardinal Stefan Wyszynski Institute of Cardiology, Warsaw, Poland
}

Adv Interv Cardiol 2019; 15, 3 (57): 308-313

DOI: https://doi.org/10.5114/aic.2019.84475

\begin{abstract}
A bstract
Introduction: Growing role of coronary computed tomography angiography (CTA) as a diagnostic tool in patients with suspected coronary artery disease (CAD) calls for better recognition of its value in clinical decision making as compared to the gold standard of invasive coronary angiography (ICA).

Aim: To assess the diagnostic value of quantitative coronary computed tomography angiography (QCT) as compared to quantitative coronary angiography (QCA) for the prediction of coronary revascularization.

Material and methods: In this prospective observational study we included 100 patients who underwent ICA following CTA. Quantitative diameter stenosis analysis (qCTA) was performed with Syngo.via (Siemens Medical Systems) software by an experienced investigator blinded to results of ICA. Quantitative Coronary Angiography (QCA) was chosen to define \%DS in a repetitive manner. ICA images were submitted to Qangio XA (Medis, Leiden, The Netherlands) software for QCA analysis.

Results: Eighty out of 400 analysed vessels were revascularized. Per-vessel diagnostic accuracy, sensitivity, specificity, PPV an NPV were $80 \%, 98 \%, 73 \%, 48 \%$ and $99 \%$ for QCT and $81 \%, 99 \%, 73 \%, 48 \%$ and $100 \%$ for QCA, respectively, for the prediction of revascularization. AUC was similar: 0.88 for QCT and 0.89 for QCA ( $p=\mathrm{NS})$.

Conclusions: These real-world data support the concept that CTA is as precise in prediction of coronary revascularization as ICA. This may add to the discussion about CTA having the potential to replace ICA for diagnosing vessels qualified for intervention, reserving the invasive diagnostic approach for those with the highest probability of revascularization.
\end{abstract}

Key words: coronary angiography, coronary computed tomography angiography.

Su m m a ry

Computed tomography angiography (CTA) is as precise in prediction of coronary revascularization as invasive coronary angiography (ICA). This may add to the discussion about CTA having the potential to replace ICA for diagnosing vessels qualified for intervention, reserving the invasive diagnostic approach for those with the highest probability of revascularization.

\section{Introduction}

Coronary computed tomography angiography (CTA) has high diagnostic accuracy in ruling out significant stenosis of coronary arteries in patients with intermediate probability of coronary artery disease (CAD) [1-3]. Moreover, provided good image quality, CTA may also accurately assess previously implanted coronary stents suspected of in-stent restenosis [4, 5]. By definition, some of the patients examined by CTA have diseased coronary arteries and undergo further diagnostic testing, including invasive coronary angiography (ICA), and may undergo revascularization, either by percutaneous coronary intervention $(\mathrm{PCl})$ or coronary artery bypass grafting (CABG). Percent diameter stenosis (\%DS) assessed by ICA plays a major role in clinical decision-making regarding coronary revascularization, as reflected in current European Society of Cardiology revascularization guidelines and clinical practice [6]. Importantly, recent studies suggest an increasing role of coronary CTA in evaluation of patients with higher (>50\%) pre-test probability of CAD, or

\section{Corresponding author:}

Mariusz Dębski MD, Department of Coronary and Structural Heart Diseases, The Cardinal Stefan Wyszynski Institute of Cardiology, 42 Alpejska St, 04-628, Warsaw, Poland, phone: +48 692577 462, e-mail: mdebski@ikard.pl

Received: 15.02.2019, accepted: 18.03.2019. 
blatant indications for invasive angiography [7, 8]. The evolving clinical landscape for use of CTA calls for better recognition of its value in clinical decision making as compared to the gold standard of ICA.

\section{Aim}

The objective of the present study was to assess the diagnostic value of quantitative coronary computed tomography angiography (QCT) as compared to quantitative coronary angiography (QCA) for the prediction of coronary revascularization.

\section{Material and methods}

\section{Study group}

From 09.2015 to 08.2016 we included 116 consecutive patients who underwent ICA following CTA performed at a single center in Warsaw, Poland. ICA was performed if CTA findings suggested significant or borderline coronary artery stenosis (>50\% DS, evaluated visually by experienced observer) in an artery amenable to intervention (at least $2.0 \mathrm{~mm}$ reference diameter), in the presence of clinical symptoms suggestive of CAD or additional tests indicating cardiac ischemia. Excluded from this group were patients $(n=10)$ who underwent ICA more than 6 months after CTA and those $(n=6)$ in whom CTA image quality prevented evaluation of the coronary artery lumen due to motion artifacts or severe calcification. Clinical and demographic information, medical history, and cardiovascular risk factors (hypertension, hyperlipidemia, diabetes, body mass index, smoking, being male) were prospectively collected. The local ethics committee approved this study.

\section{CTA examination and analysis}

Coronary CTA was performed on a dual source $2 \times$ 192-slice Somatom Force (Siemens, Forchheim, Germany) scanner. Sublingual nitrates were administered prior to scanning in all patients. If necessary, $\beta$-blockers were administered intravenously targeting a heart rate $<70$ beats per minute. The protocol for CTA image acquisition was recommended to comply with the Society of Cardiovascular Computed Tomography (SCCT) guidelines [9]. Assessment of luminal diameter stenosis was performed using an 18-segment coronary model. Quantitative diameter stenosis analysis (QCT) was performed with Syngo.via (Siemens Medical Systems) software by an experienced investigator blinded to the results of ICA. The intraobserver correlation coefficient performed in 60 randomly chosen vessels was 0.99 (95\% Cl: 0.98-0.99, $p<0.0001$ for correlation). Per-vessel maximum stenosis was categorized as 0\%, 1-24\%, 25-49\%, 50-69\%, 7099\%, 100\% according to SCCT guidelines [10]. Calcium score was calculated according to the Agatston method. Additionally, lesions were divided into non-calcified (no calcification), mixed (some calcification) and calcified (massive calcification) based on visual assessment.

\section{ICA examination and angiographic analysis}

The ICAs were performed on a standard cardiology fluoroscopy equipment (Axiom, Siemens Healthcare, Forchheim, Germany), in pulsed fluoroscopy mode with a default frame rate of 10 frames per second. Access site and utilization of additional tools (i.e. fractional flow reserve (FFR) assessment or intravascular ultrasound (IVUS)) was left to the discretion of the operator. Therapy decision was made on the basis of angiographic results in the context of the patient's symptoms and other test results, such as stress ECG or echocardiography. Data from the literature suggest that visual vessel assessment during ICA is highly subjective $[11,12]$, so quantitatve coronary angiography (QCA) was chosen to define \%DS in a repetitive manner. ICA images were submitted to Qangio XA (Medis, Leiden, The Netherlands) software for QCA analysis. Maximum diameter stenosis was automatically defined with subsequent manual alignment of the course of the vessel, if necessary. Per-vessel maximum stenosis was categorized as 0\%, 1-24\%, 25-49\%, 50-69\%, 70-99\%, 100\%. The analyses were performed for the right coronary artery (RCA), left main (LM), left anterior descending (LAD) and circumflex branch (CX).

\section{Statistical analysis}

The categorical variables are presented as numbers and percentages. The continuous variables are expressed as mean \pm SD or median $\left(25^{\text {th }}-75^{\text {th }}\right.$ percentile $)$ as appropriate. Descriptive statistics were used to analyze per-vessel accuracy of CTA and QCA. The diagnostic performance of CTA and QCA in the prediction of revascularization was presented as accuracy, sensitivity, specificity, positive predictive value (PPV) and negative predictive value (NPV) parameters and their corresponding 95\% confidence intervals (Cls), as well as by receiver operator characteristics (ROC) analysis comparison. Paired samples $t$-tests or Wilcoxon test (as appropriate) were used to assess the equality of means in continuous variables. QCA and QCT comparison, including assessing lesions according to their calcification, was performed with the Bland-Altman test. A probability value of 0.05 or less was considered significant. All statistical analyses were conducted using MedCalc version 13.0 (MedCalc Software, Mariakerke, Belgium).

\section{Results}

In this study, 116 consecutive patients who underwent CTA and subsequently ICA were identified. After applying exclusion criteria, the final population consisted of 100 patients in whom 400 vessels (RCA, LM, LAD, Cx) were assessed. Baseline characteristics of the population (mean age: $67.1 \pm 8.8$, female $33 \%$ ) are summarized in 
Table I. The majority of patients (54\%) presented with typical angina. In this group Canadian Cardiovascular Society grade 2 was most commonly observed. Thirty-nine patients had undergone stress tests before coronary imaging - 24 tests had been positive electrocardiographically, 6 clinically, 3 had been inconclusive and 6 negative. Among 17 patients with $\mathrm{PCl}$ history, all stents were imaged by CTA without significant blooming artifacts precluding stent patency evaluation. During ICA, either FFR $(n=11$, mean result 0.82$)$ or IVUS $(n=5$, mean MLA $5 \mathrm{~mm}^{2}$ ) was used in 16 cases. Mean time interval between CTA and ICA was $60.3 \pm 50$ days. No serious adverse events were observed during either CTA or ICA.

Based on ICA findings $\mathrm{PCl}$ was performed in 53 patients, and 11 patients underwent subsequent coronary artery bypass grafting (CABG). Ultimately, 80 vessels were revascularized.

Median calcium score per patient was 424 (95\% Cl: 296-594). Mean stenoses of RCA, LM, LAD and Cx as assessed by QCA were $52 \%, 12 \%, 56 \%$ and $40 \%$, respectively, and by QCT 50\%, 11\%, 59\% and 40\%, respectively. Per-vessel diagnostic accuracy, sensitivity, specificity, PPV an NPV were $80 \%, 98 \%, 73 \%, 48 \%$ and $99 \%$ for QCT and $81 \%, 99 \%, 73 \%, 48 \%$ and $100 \%$ for QCA, respectively, for prediction of revascularization (Figure 1). An example of QCT and QCA lesion analysis is presented in Figure 2. Bland-Altman plots assessing agreement between the two methods in all lesions, in non-calcified lesions, in mixed lesions and in stents are presented in Figures 3-6, respectively. AUC for predicting revascularization was similar: 0.88 for QCT and 0.89 for QCA $(p=N S)$. No significant differences were found on an individual vessel level: AUC for the left anterior descending artery 0.79 and 0.79 , for the circumflex artery 0.89 and 0.89 , for the right coronary artery 0.84 and 0.85 , for QCT and QCA respectively. Both methods performed similarly regardless of le-

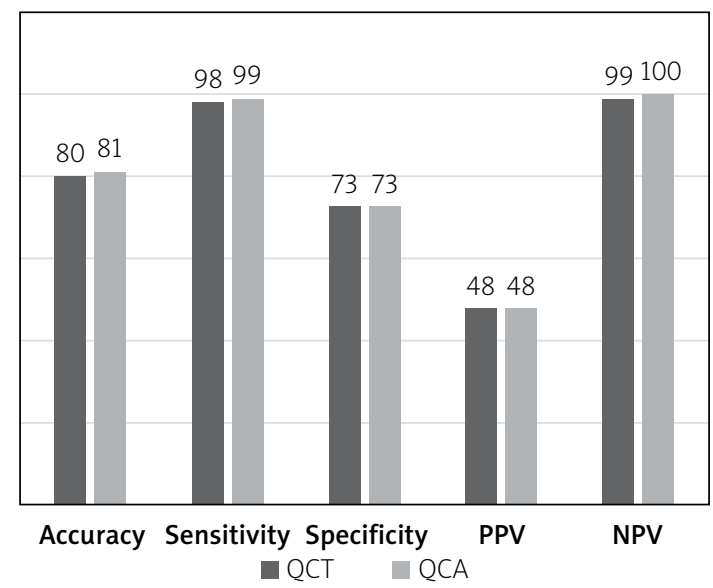

Figure 1. Per-vessel diagnostic performance of both cardiac CT scans and invasive angiography

Both tests demonstrated similar diagnostic accuracy for detecting vessels that eventually were revascularized. QCT - quantitative coronary computed tomography, PPV - positive predictive value, NPV negative predictive value, QCA - quantitative coronary angiography.
Table I. Baseline patients' characteristics and clinical assessment $(n=100)$

\begin{tabular}{|c|c|}
\hline Parameter & Result \\
\hline Age, mean \pm SD [years] & $67.1 \pm 8.8$ \\
\hline Male gender, $n=\%$ & 67 \\
\hline Height, mean \pm SD $[\mathrm{m}]$ & $1.70 \pm 0.09$ \\
\hline Body weight, mean \pm SD $[\mathrm{kg}]$ & $82.1 \pm 13.2$ \\
\hline Body mass index, mean $\pm \mathrm{SD}\left[\mathrm{kg} / \mathrm{m}^{2}\right]$ & $28.5 \pm 4.15$ \\
\hline Hypertension, $n=\%$ & 91 \\
\hline Diabetes mellitus, $n=\%$ & 32 \\
\hline Hyperlipidemia, $n=\%$ & 89 \\
\hline Smoking history, $n=\%$ & 68 \\
\hline Pack-years, mean \pm SD [years] & $17.8 \pm 20.0$ \\
\hline Current smoker, $n=\%$ & 15 \\
\hline Ejection fraction, mean $\pm \mathrm{SD}(\%)^{*}$ & $61.1 \pm 8.1$ \\
\hline Atypical angina, $n=\%$ & 46 \\
\hline Typical angina, $n=\%$ & 54 \\
\hline $\operatorname{CCS} 1$ & $7 / 54(13.0)$ \\
\hline $\operatorname{ccs} 2$ & $28 / 54(51.9)$ \\
\hline $\operatorname{cCS} 3$ & $16 / 54(29.6)$ \\
\hline $\operatorname{cCS} 4$ & $3 / 54(5.6)$ \\
\hline Chronic kidney disease, $n=\%$ & 35 \\
\hline $\mathrm{PCl}$ history, $n=\%$ & 17 \\
\hline CABG history, $n=\%$ & 6 \\
\hline AMI history, $n=\%$ & 13 \\
\hline Family history of CAD, $n=\%$ & 28 \\
\hline \multicolumn{2}{|l|}{ Stress electrocardiograph: } \\
\hline Performed, $n=\%$ & 39 \\
\hline Clinically positive, $n(\%)$ & $6 / 39(15.4)$ \\
\hline ECG-positive, $n(\%)$ & $24 / 39(61.5)$ \\
\hline Negative, $n(\%)$ & $6 / 39(15.4)$ \\
\hline Inconclusive, $n(\%)$ & $3 / 39(7.7)$ \\
\hline Serum total cholesterol, mean \pm SD [mmol/l]: & $4.3 \pm 1.1$ \\
\hline Low-density lipoprotein, mean \pm SD [mmol/l] & $2.5 \pm 0.9$ \\
\hline High-density lipoprotein, mean \pm SD [mmol/l] & $1.4 \pm 0.4$ \\
\hline Statin, $n=\%$ & 90 \\
\hline ACE-inhibitor or ARB, $n=\%$ & 86 \\
\hline Calcium channel blocker, $n=\%$ & 44 \\
\hline$\beta$-Blockade, $n=\%$ & 85 \\
\hline Acetylsalicylic acid use, $n=\%$ & 100 \\
\hline
\end{tabular}

CCS - Canadian Cardiovascular Society, $P C I$ - percutaneous coronary intervention, $C A B G$ - coronary artery bypass grafting, $A M I$ - acute myocardial infarction, $C A D$ - coronary artery disease, ECG - electrocardiography, $A C E$ - angiotensin converting enzyme, $A R B$ - angiotensin II receptor blocker. * Data available for 81 patients. 

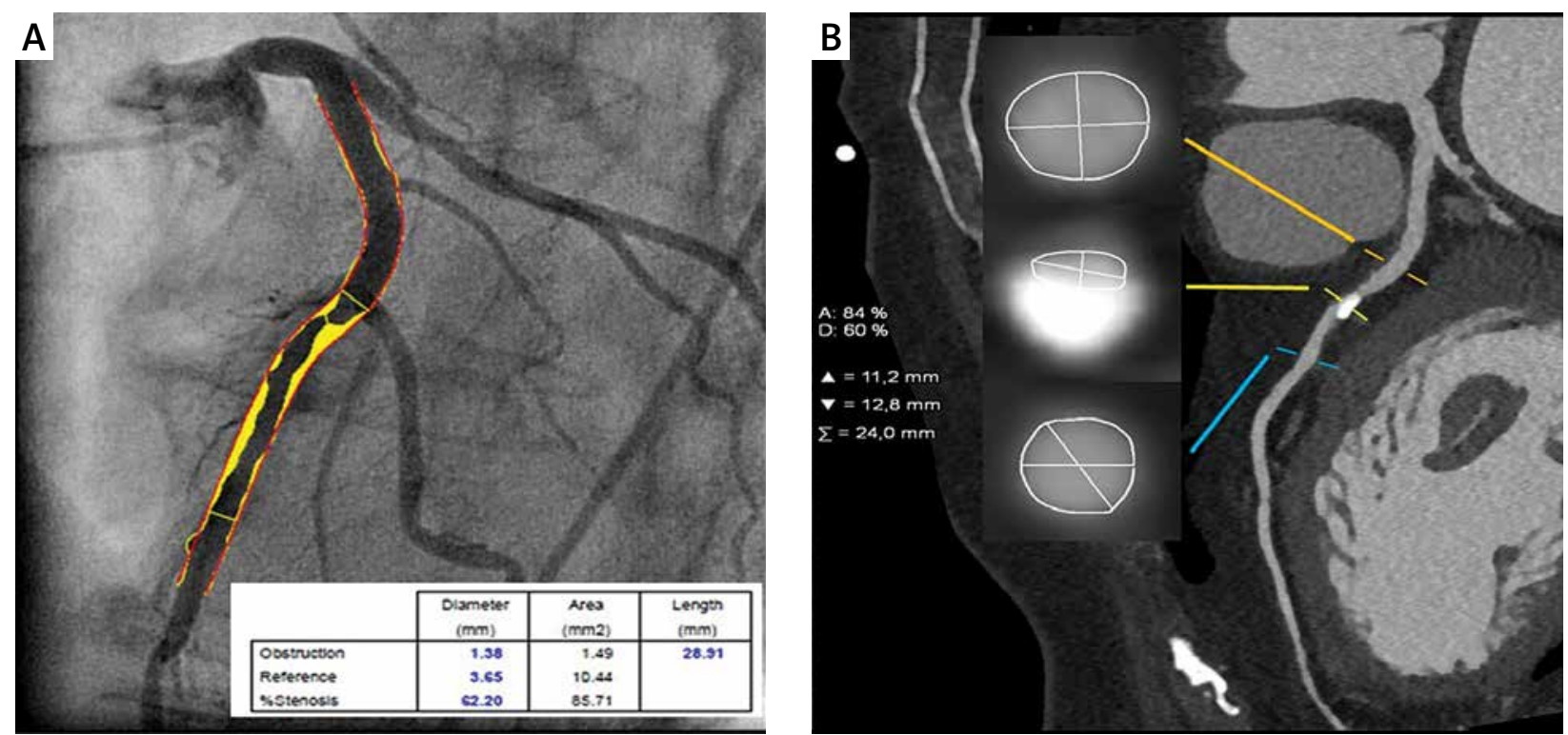

Figure 2. Lesion analysis in quantitative coronary angiography (A) and quantitative coronary computed tomography (B)

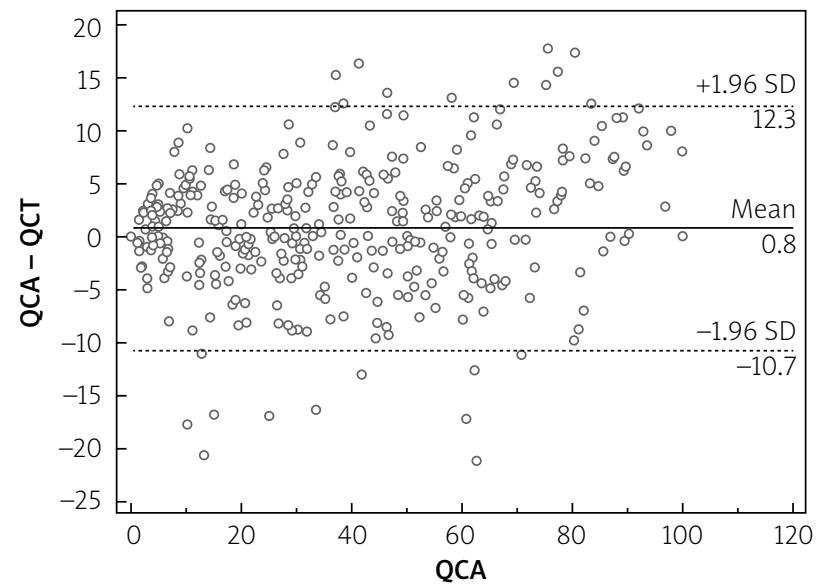

Figure 3. Bland-Altman plot comparing QCA and QCT results (all lesions)

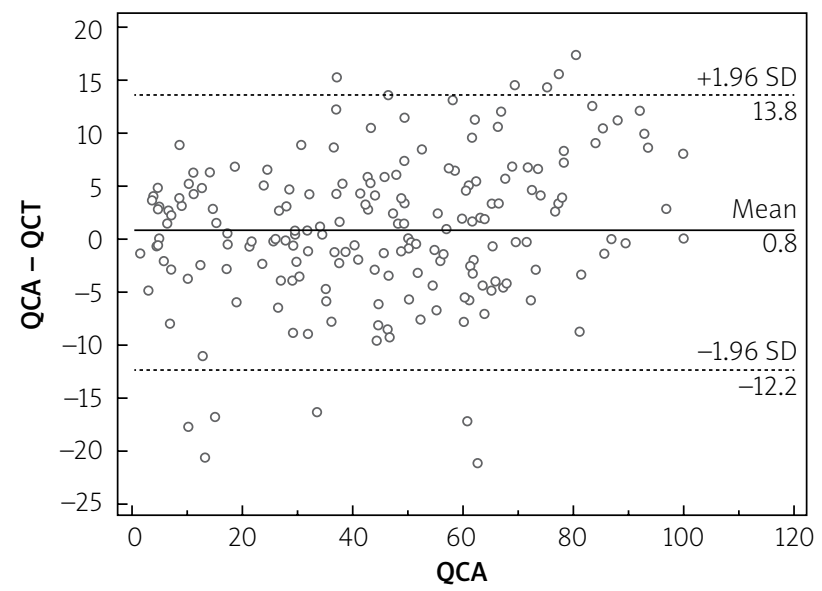

Figure 5. Bland-Altman plot comparing QCA and QCT results (mixed lesions)

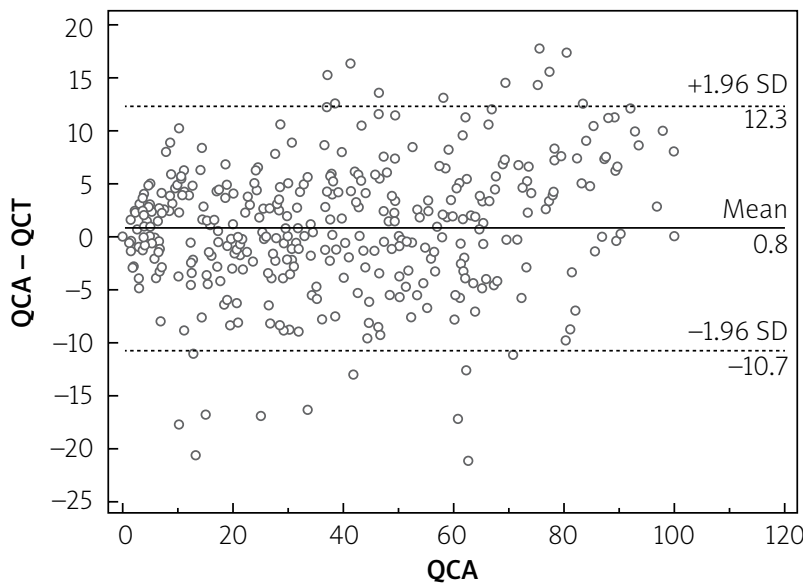

Figure 4. Bland-Altman plot comparing QCA and QCT results (non-calcified lesions)

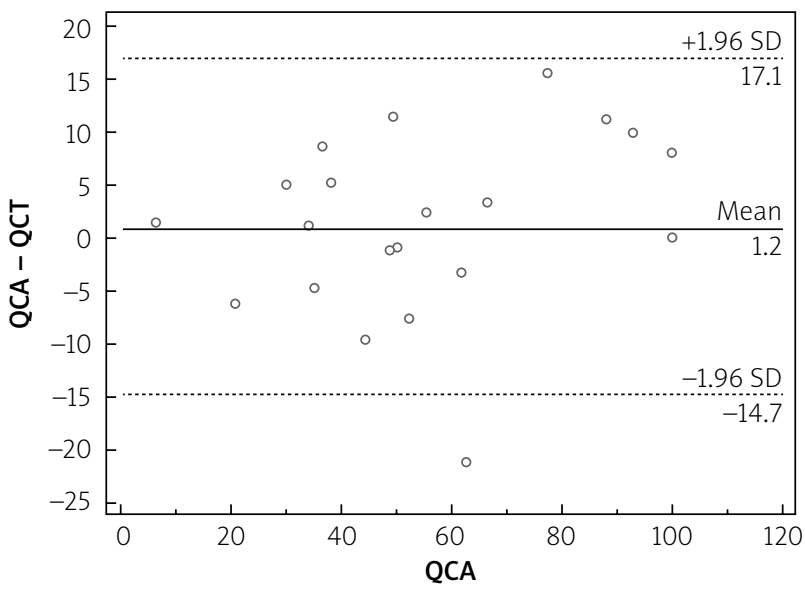

Figure 6. Bland-Altman plot comparing QCA and QCT results (stents) 
sion calcification - AUC in non-calcified lesions $(n=193)$ for QCA was 0.957 (95\% Cl: 0.918-0.981) and for QCT 0.955 (95\% Cl: 0.916-0.980), whereas in mixed lesions $(n=201)$ AUC for QCA was 0.833 (95\% Cl: 0.774-0.881) and for QCT 0.825 (95\% Cl: 0.765-0.875). The reduction in AUC in mixed lesions compared to non-calcified lesions was statistically significant for both methods ( $p<0.001$ for both). Calcified lesions were too infrequent to warrant valid statistical analysis $(n=7)$. Considering previously implanted stents' assessment $(n=23)$, further reduction in AUC (although not statistically significant as compared to mixed lesions) was observed, yet again comparable between the two methods $-0.676(95 \% \mathrm{Cl}$ : $0.451-0.854)$ for QCA and 0.672 (95\% Cl: 0.447-0.851).

\section{Discussion}

The CTA, as a non-invasive diagnostic method, is recommended for patients with intermediate CAD probability [13] and is widely used as first-line diagnostics in patients with suspected CAD. Recent studies suggest that the method can be effectively used in patients with direct indications for invasive angiography $[7,8]$. The expanding diagnostic role of coronary CTA calls for better recognition of its capacity to provide the basis for clinical decision making. We found that the predictive accuracy of coronary CTA-derived measurements of stenosis severity had similar diagnostic accuracy compared with QCA in prediction of coronary revascularization, which was impacted neither by presence of calcifications nor by stents. This finding suggests that, due to lower costs and improved safety, coronary CTA may act as a gatekeeper and first-line test to triage patients to medical therapy or invasive evaluation with potential revascularization. The high specificity and NPV of CTA are comparable with previous studies [1, 2]. The relatively low PPV of both methods is expected and related to the fact that not all lesions with stenosis $>50 \%$ in diameter necessitate intervention, as other factors (i.e. clinical symptoms or FFR measurement) influence the revascularization decision [14]. Our data are consistent with three papers from recent years, in which measurements derived from CTA performed similarly to QCA-derived measurements in assessing luminal stenosis, at both a per-patient and per-vessel level [15-17]. Our results are also in line with previously reported, slightly lower accuracy of CTA in lesions with calcifications [18] or previously implanted stents [4, 5]; however, in our dataset also QCA performed worse in such conditions. Furthermore, in the mentioned studies CTA findings accurately evaluated ischemia-causing lesions, with invasive FFR as a reference standard, which is accordant with our conclusion about CTA ability to predict coronary revascularization. Additionally, besides anatomical assessment provided by CTA, functional assessment became recently feasible with CTA-derived FFR. It emerged as a promising, non-invasive diagnostic tool, whose main advantage over plain coronary CTA is improved specificity for diagnosing functionally significant stenosis $[19,20]$.

\section{Study limitations}

We acknowledge several limitations of the current study. The sample size is limited and is derived from a single center. The study was conceived as an observational, non-randomized project, based on prospectively collected data. Non-randomization and the fact that several operators performed ICAs may have influenced procedural aspects such as use of additional tools (i.e. FFR, IVUS) and therefore may have, to some extent, affected revascularization decisions. This, however, reflects everyday practice.

Recognizing its inherent limitations, the study does have the advantage of representing the daily practice in a diverse patient population, as no clinical exclusion criteria were applied.

\section{Conclusions}

These real-world data support the concept that CTA is as precise in prediction of coronary revascularization as ICA. This may add to the discussion about CTA having the potential to replace ICA for diagnosing vessels qualified for intervention, reserving the invasive diagnostic approach for those with the highest probability of revascularization.

\section{Acknowledgments}

Dr Dębski and Dr Kruk equally contributed to the paper.

\section{Conflict of interest}

The authors declare no conflict of interest.

\section{References}

1. Raff GL, Gallagher MJ, O'Neill WW, Goldstein JA. Diagnostic accuracy of noninvasive coronary angiography using 64-slice spiral computed tomography. J Am Coll Cardiol 2005; 46: 552-7.

2. Newby D. CT coronary angiography in patients with suspected angina due to coronary heart disease (SCOT-HEART): an open-label, parallel-group, multicentre trial. Lancet 2015; 385: 2383-91.

3. Shaw LJ, Hausleiter J, Achenbach S, et al. Coronary computed tomographic angiography as a gatekeeper to invasive diagnostic and surgical procedures: results from the multicenter confirm (coronary ct angiography evaluation for clinical outcomes: an international multicenter) registry. J Am Coll Cardiol 2012; 60: 2103-14.

4. Rixe J, Achenbach S, Ropers D, et al. Assessment of coronary artery stent restenosis by 64 -slice multi-detector computed tomography. Eur Heart J 2006; 27: 2567-72.

5. Carbone I, Francone M, Algeri E, et al. Non-invasive evaluation of coronary artery stent patency with retrospectively ECG-gated 64-slice CT angiography. Eur Radiol 2008; 18: 234-43.

6. Neumann FJ, Sousa-Uva M, Ahlsson A, et al. 2018 ESC/EACTS Guidelines on myocardial revascularization. Eur Heart J 2019; 40: $87-165$ 
7. Rudziński PN, Kruk M, Kępka C, et al. The value of coronary artery computed tomography as the first-line anatomical test for stable patients with indications for invasive angiography due to suspected coronary artery disease: CAT-CAD randomized trial. J Cardiovasc Comput Tomogr 2018; 12: 472-9.

8. Chang HJ, Lin FY, Gebow D, et al. Selective referral using CCTA versus direct referral for individuals referred to invasive coronary angiography for suspected CAD. JACC Cardiovasc Imaging 2019; 12: 1303-12.

9. Abbara S, Blanke P, Maroules CD, et al. SCCT guidelines for the performance and acquisition of coronary computed tomographic angiography: a report of the Society of Cardiovascular Computed Tomography Guidelines Committee Endorsed by the North America. J Cardiovasc Comput Tomogr 2016; 10: 435-49.

10. Raff GL, Abidov A, Achenbach S, et al. SCCT guidelines for the interpretation and reporting of coronary computed tomographic angiography. J Cardiovasc Comput Tomogr 2009; 3: 122-36.

11. Nallamothu BL, Spertus JA, Lansky AJ, et al. Comparison of clinical interpretation with visual assessment and quantitative coronary angiography in patients undergoing percutaneous coronary intervention in contemporary practice: the assessing angiography (A2) Project. Circulation 2013; 151: 414-20.

12. Zhang H, Mu L, Hu S, et al. Comparison of physician visual assessment with quantitative coronary angiography in assessment of stenosis severity in China. JAMA Intern Med 2018; 178: 239-47.

13. Montalescot G. Guidelines on the management of stable coronary artery disease. Eur Heart J 2014; 69: 51-2.

14. Adjedj J, Xaplanteris P, Toth G, et al. Visual and quantitative assessment of coronary stenoses at angiography versus fractional flow reserve: the impact of risk factors. Circ Cardiovasc Imaging 2017; 10:pii: e006243.

15. Budoff MJ, Nakazato R, Mancini GBJ, et al. CT angiography for the prediction of hemodynamic significance in intermediate and severe lesions head-to-head comparison with quantitative coronary angiography using fractional flow reserve as the reference standard. JACC Cardiovasc Imaging 2016; 9: 559-64.

16. Nørgaard BL, Leipsic J, Gaur S, et al. Diagnostic performance of noninvasive fractional flow reserve derived from coronary computed tomography angiography in suspected coronary artery disease: the NXT trial (Analysis of Coronary Blood Flow Using CT Angiography: Next Steps). J Am Coll Cardiol 2014; 63: 1145-55.

17. Voros S, Rinehart S, Vazquez-Figueroa JG, et al. Prospective, head-to-head comparison of quantitative coronary angiography, quantitative computed tomography angiography, and intravascular ultrasound for the prediction of hemodynamic significance in intermediate and severe lesions, using fractional flow reserve as reference standard (from the ATLANTA $\mathrm{i}$ and II Study). Am J Cardiol 2014; 113: 23-9.

18. Kruk M, Noll D, Achenbach S, et al. Impact of coronary artery calcium characteristics on accuracy of CT angiography. JACC Cardiovasc Imaging 2014; 7: 49-58.

19. Sand NPR, Veien KT, Nielsen SS, et al. PRrospEctive Comparison of FFR Derived From Coronary CT Angiography With SPECT PerfuSion Imaging in Stable Coronary ArtEry DiSeaSe: The ReASSESS Study. JACC Cardiovasc Imaging 2018; 11: 1640-50.

20. Kruk M, Wardziak Ł, Demkow M, et al. Workstation-based calculation of CTA-based FFR for intermediate stenosis. JACC Cardiovasc Imaging 2016; 9: 690-9. 